\title{
Antimicrobial properties of hydrogen peroxide and potash alum alone and in combination against clinical bacterial isolates
}

\author{
Huma Khurshid ${ }^{1}$, Muhammad Rafiq ${ }^{2}$, Fahad Nazir ${ }^{3}$, Imran $\mathrm{Ali}^{4}$, \\ Muneer Ahmed ${ }^{5}$, Bilal Akbar ${ }^{6}$, Mushtaq Ahmed ${ }^{1}$ and Anser Ali ${ }^{*}$ \\ 1. Department of Zoology, Mirpur University of Science and Technology (MUST), Mirpur-10250 (AJK)- \\ Pakistan \\ 2. Department of Physiology \& Biochemistry, Cholistan University of Veterinary \& Animal Sciences, \\ Bahawalpur-Pakistan \\ 3. Department of Entomology, Faculty of Crop Protection, Sindh Agriculture University Tandojam-Pakistan \\ 4. Department of Biotechnology, Mirpur University of Science and Technology (MUST), Mirpur-10250 (AJK)- \\ Pakistan \\ 5. School of Biological Sciences, University of Punjab, Quaid-e-Azam Campus, Lahore-Pakistan \\ 6. Department of Mechanical Engineering, Mirpur University of Science and Technology (MUST), Mirpur- \\ 10250 (AJK)-Pakistan \\ *Corresponding author's email: anser.zoology@must.edu.pk \\ Citation \\ Huma Khurshid, Muhammad Rafiq, Fahad Nazir, Imran Ali, Muneer Ahmed, Bilal Akbar, Mushtaq Ahmed and \\ Anser Ali. Antimicrobial properties of hydrogen peroxide and potash alum alone and in combination against \\ clinical bacterial isolates. Pure and Applied Biology. Vol. 8, Issue 4, pp2238-2247. \\ http://dx.doi.org/10.19045/bspab.2019.80169
}

\begin{tabular}{llll}
\hline \hline Received: $20 / 05 / 2019$ & Revised: 16/07/2019 & Accepted: 24/07/2019 & Online First: 03/08/2019 \\
\hline
\end{tabular}

\section{Abstract}

Hydrogen peroxide $\left(\mathrm{H}_{2} \mathrm{O}_{2}\right)$ and potash alum (ALM) are considered important for wide range of biological activities including microbial disinfection. However, comparison of $\mathrm{H}_{2} \mathrm{O}_{2}$ and ALM antibacterial effect alone and in combination on different clinical bacterial isolates with respect to post treatment time is still not well studied. Thus, in present study we tested susceptibility of five bacterial isolates; Enterococcus faecalis (E. faecalis), Enterococcus faecium (E. faecium), Escherichia coli (E. coli), Staphylococcus aureus (S. aureus) and Klebsiella pneumoniae (K. pneumonia) against $\mathrm{H}_{2} \mathrm{O}_{2}$ and ALM alone and in combination which showed concentration dependent and incubation dependent effect. The $\mathrm{H}_{2} \mathrm{O}_{2}$ bacterial susceptibility trend was $E$. coli $>K$. pneumoniae $>S$. aureus $>E$. faecium $>$ E. faecalis and ALM susceptibility trend was as $K$. pneumoniae $>S$. aureus $>$ E. faecalis $>$ E. coli $>$ E. faecium at highest tested concentration $(35 \mathrm{mg} / \mathrm{ml})$ and $24 \mathrm{hr}$ of incubation period. Comparatively, antimicrobial activity was higher with $\mathrm{H}_{2} \mathrm{O}_{2}$ than ALM however in the order of $24 \mathrm{hr}>48 \mathrm{hr}>72 \mathrm{hr}$. Both $\mathrm{H}_{2} \mathrm{O}_{2}$ and ALM showed more zones of inhibition at highest tested concentration than positive control azithromycin (AZI) against $E$. faecalis, E. faecium and $S$. aureus at all incubation periods. Moreover, $\mathrm{H}_{2} \mathrm{O}_{2}$ and ALM in combination (1:1) showed increased zone of inhibition than ALM alone (against all bacteria), $\mathrm{H}_{2} \mathrm{O}_{2}$ alone (against E. faecalis and E. faecium) and, AZI (against E. faecalis, E. faecium and $S$. aureus) without increasing final concentration. Thus, combination treatment might be more effective disinfection and antisepsis strategy which may help us in minimizing dose dependent side effects without compromising efficacy.

Keywords: Combination; Hydrogen peroxide; Potash alum; Zones of inhibition 


\section{Introduction}

Antimicrobial agents are extensively utilized in health care, industry and the environment to control and treat microbial infection [1, 2]. Combining antimicrobial agent may improve their activities by synergism and may provide an effective alternative to microbial resistance against single chemical [1, 3]. Additionally, a combination strategy is cost effective and time-saving which does not require long laboratory work to design, synthesize or discover new drugs more frequently. Thus, encouraging the use of drugs in combination for the management of bacterial infections.

Hydrogen peroxide $\left(\mathrm{H}_{2} \mathrm{O}_{2}\right)$, our study compound, retains bactericidal efficacy which is being observed in $\mathrm{H}_{2} \mathrm{O}_{2}$ producing bacteria and phagocytic cells that inhibit other bacterial species and can kill invading microorganism, respectively [4]. The ability of hydrogen peroxide to produce hydroxyl radical contributes in the oxidation process of biomolecules. Hydrogen peroxide conversion to cytotoxic compounds is supported by peroxidases and by reducing agents [4]. Hydrogen peroxide along with produced free hydroxyl radicals may contribute in microbial management [1]. $\mathrm{H}_{2} \mathrm{O}_{2}$ is common disinfectant used to control gingival plaques [5], treating biofilms [6] and Fournier's gangrene [7]. It is also used for the sterilization of other surfaces [8] including industrial fish egg $[9,10]$ and fruits treatments [11].

Alum (ALM), our second study compound is a salt known as aluminium potassium sulfate $\left(\mathrm{KAl}\left(\mathrm{SO}_{4}\right)_{2}\right)$ [12] and is suggested as a class 1 dynamic compound in mouthwashes [13], and used for the treatment of pediatric cough, hemorrhagic cystitis [14] ulcers, oral cavities and burns with anticarcinogenic effect [15], cosmetics [ 16], and domestic and industrial water treatments [17]. It has potential inhibitory effects on microbes [18].
In infection treatment, side effects associated with an overload of drugs in the human body and reduced microbial susceptibility to a single drug could be controlled by using them in combination even at lower concentrations. A synergistic or additive effect in mixtures is a key point in decreasing concentrations of drugs without compromising outcomes. Similarly, the synergy of hydrogen peroxide was found with many compounds including rifampicin [19], chlorhexidine [20], neucoproine [21], hypothiocyanite [22], sodium bicarbonate [23], iodine [2426], different organic acids $[27,28]$, as well as with UV-irradiation [29]. Likewise, alum in combination with leaf extract of guava (Psidium guajava) has been utilized topically as an antiperspirant, an antibacterial and for astringent purposes [30]. However, alum and $\mathrm{H}_{2} \mathrm{O}_{2}$ incubation wise and concentration wise in combination against various microorganisms were not studied. Thus, in this study, we compared incubationdependent and concentration-dependent inhibitory effects of alum and hydrogen peroxide alone and in mixtures against five clinical bacterial isolates.

\section{Materials and methods}

\section{Culture media and chemicals}

Nutrient agar (Neogen Company, UK), nutrient broth (Merck, Germany), and dimethyl sulfoxide (BIOCHEM) were used in experiments. Test compounds hydrogen peroxide (Sigma) and potash alum (purchased from the local market of Mirpur, AJK) were diluted with saline to acquire desired concentrations.

\section{Strains and culture conditions}

The clinical bacterial isolates Enterococcus faecalis (E. faecalis), Enterococcus faecium (E. faecium), Escherichia coli (E. coli), Staphylococcus aureus (S. aureus) and Klebsiella pneumoniae ( $K$. pneumonia) were taken from the Combined Military Hospital (CMH) Rawalpindi (Pakistan) for this study. Bacteria were sub-cultured on 
nutrient agar plates and incubated at $37^{\circ} \mathrm{C}$ for $24 \mathrm{hr}$ in this experiment.

Anti-bacterial activity of alum and hydrogen peroxide separately

Fresh bacterial cultures were prepared by spreading $70 \mu \mathrm{l}$ bacterial suspension $(0.060$ $\mathrm{OD}$ at 600 wavelengths) on agar plate homogeneously. After short air dry (10min), 4 wells (6mm diameter) were made on each plate and loaded with $50 \mu 1$ of test compounds having $4.4 \mathrm{mg} / \mathrm{ml}$, $8.7 \mathrm{mg} / \mathrm{ml}, \quad 17.5 \mathrm{mg} / \mathrm{ml}$ and $35 \mathrm{mg} / \mathrm{ml}$ concentrations, respectively. Later, the plates were incubated at $37^{\circ} \mathrm{C}$ for $24 \mathrm{hr}$, $48 \mathrm{hr}$ and $72 \mathrm{hr}$. Finally, plates were photographed and zones of inhibition were measured in $\mathrm{cm}$.

Anti-bacterial activity of alum and hydrogen peroxide in combination

In combination test, alum and hydrogen peroxide were used in 1:1 ratio with twofold increasing concentration from well-1 to well-4, respectively. Briefly, well-1, well-2, well-3 and well-4 were loaded with $50 \mu 1$ of alum and hydrogen peroxide in $1: 1$ ratio from $4.4 \mathrm{mg} / \mathrm{ml}, \quad 8.7 \mathrm{mg} / \mathrm{ml}$, $17.5 \mathrm{mg} / \mathrm{ml}$ and $35 \mathrm{mg} / \mathrm{ml}$ concentrations for testing their synergistic effects. Later, the plates were incubated, photographed and zones of inhibition were measured the same as in the screening of alum and hydrogen peroxide separately.

Statistical Analysis

All experiments were performed thrice in triplicates. Student's t-test was applied to check significant differences using the lowest zone of inhibition (except zero) VS each tested zone of inhibition $\left({ }^{\mathrm{a}} \mathrm{p}<0.05\right.$; $\left.{ }^{b} \mathrm{p}<0.005,{ }^{c} \mathrm{p}<0.0001\right)$.

\section{Results and Discussion}

The present study was carried out to evaluate the antibacterial activity of hydrogen peroxide $\left(\mathrm{H}_{2} \mathrm{O}_{2}\right)$ and potash alum (ALM) alone and in combination. For this, well diffusion method was used against five pathogenic bacterial isolates namely E. coli, E. faecium, E. faecalis, $S$. aureus and $K$. pneumoniae. Later, zones of inhibition were measured as a representative of bacterial susceptibility at different concentrations and different incubation periods at $24 \mathrm{hr}, 48 \mathrm{hr}$ and $72 \mathrm{hr}$.

\section{Individual antibacterial effect of alum} and hydrogen peroxide

The hydrogen peroxide tested concentrations, $\quad 35 \mathrm{mg} / \mathrm{ml}, \quad 17.5 \mathrm{mg} / \mathrm{ml}$, $8.7 \mathrm{mg} / \mathrm{ml}$ and $4.4 \mathrm{mg} / \mathrm{ml}$ showed $0.783 \mathrm{~cm}$, $0.693 \mathrm{~cm}, \quad 0.581 \mathrm{~cm}, \quad 0.461 \mathrm{~cm}$ zones of inhibition against E. faecalis, $0.872 \mathrm{~cm}$, $0.725 \mathrm{~cm}, 0.612 \mathrm{~cm}, 0.522 \mathrm{~cm}$ zones of inhibition against E. faecium, $0.891 \mathrm{~cm}$, $0.712 \mathrm{~cm}, 0.583 \mathrm{~cm}, 0.421 \mathrm{~cm}$ zones of the inhibition against $S$. aureus, $1.129 \mathrm{~cm}$, $0.976 \mathrm{~cm}, 0.806 \mathrm{~cm}, 0.647 \mathrm{~cm}$ zones of inhibition against $E$. coli and $0.998 \mathrm{~cm}$, $0.821 \mathrm{~cm}, 0.705 \mathrm{~cm}, 0.579 \mathrm{~cm}$ zones of inhibition against $K$. pneumoniae, respectively at $24 \mathrm{hr}$ incubation (Table 1). Hydrogen peroxide is known as an effective disinfectant and it retains the ability to quickly kill microorganisms [31]. In addition to antimicrobial activity of hydrogen peroxide because of its oxidative properties it generates more powerful oxidant, called hydroxyl radical [32]. Explaining hydrogen peroxide's mode of action, it is reported that hydrogen peroxide exposure to logarithmically growing $E$. coli to kill by damaging DNA at low concentration whereas higher concentration (> $10 \mathrm{mM}$ ) may kill by damaging all macromolecules [33-36]. 
Table 1. Antibacterial susceptibility of selected bacteria against $\mathrm{H}_{2} \mathrm{O}_{2}$, ALM and control AZI at indicated concentrations and incubation periods. Student's t-test was applied to check significant where ${ }^{\mathrm{a} p}<0.05 ;{ }^{b} \mathbf{p}<0.005,{ }^{\mathrm{c}} \mathbf{p}<0.0001$

\begin{tabular}{|c|c|c|c|c|c|c|c|c|c|c|c|c|c|}
\hline \multirow{3}{*}{ 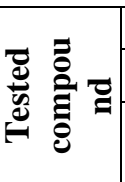 } & \multicolumn{4}{|c|}{ Concentration (mg/ml) } & \multicolumn{4}{|c|}{ Concentration $(\mathrm{mg} / \mathrm{ml})$} & \multicolumn{4}{|c|}{ Concentration (mg/ml) } & \multirow{3}{*}{ 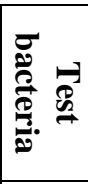 } \\
\hline & 4.4 & 8.7 & 17.5 & 35 & 4.4 & 8.7 & 17.5 & 35 & 4.4 & 8.7 & 17.5 & 35 & \\
\hline & \multicolumn{4}{|c|}{$\begin{array}{c}\text { Zone of inhibition (cm) } \\
\text { at } 24 \mathrm{hr} \text { incubation }\end{array}$} & \multicolumn{4}{|c|}{$\begin{array}{l}\text { Zone of inhibition }(\mathrm{cm}) \\
\text { at } 48 \mathrm{hr} \text { incubation }\end{array}$} & \multicolumn{4}{|c|}{$\begin{array}{l}\text { Zone of inhibition }(\mathrm{cm}) \\
\text { at } 72 \mathrm{hr} \text { incubation }\end{array}$} & \\
\hline AZI & $0.00 \pm 0.00$ & $0.09 \pm 0.02$ & $\underset{\mathrm{c}}{0.28 \pm 0.01}$ & $\underset{\mathbf{c}}{0.33 \pm 0.01}$ & $0.00 \pm 0.00$ & $\underset{\mathrm{a}}{0.09 \pm 0.02}$ & $\underset{\mathbf{b}}{0.23 \pm 0.00}$ & $0.29 \pm 0.03^{c}$ & $0.00 \pm 0.00$ & $\begin{array}{c}0.13 \pm 0.02 \\
\mathrm{a}\end{array}$ & $\underset{\mathbf{b}}{0.22 \pm 0.00}$ & $\underset{\mathbf{c}}{0.29 \pm 0.01}$ & \multirow{3}{*}{ 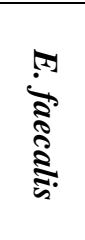 } \\
\hline $\mathrm{H}_{2} \mathrm{O}_{2}$ & $0.46 \pm 0.01$ & $\underset{\mathbf{c}}{0.58 \pm 0.01}$ & $0.69 \pm 0.01^{\mathbf{c}}$ & $\underset{\mathbf{c}}{0.78 \pm 0.01}$ & $0.46 \pm 0.01$ & $\underset{\mathbf{c}}{0.58 \pm 0.01}$ & $\underset{\mathrm{c}}{0.69 \pm 0.01}$ & $0.78 \pm 0.01^{\mathrm{c}}$ & $0.45 \pm 0.01$ & $\begin{array}{c}0.53 \pm 0.02 \\
\mathrm{a}\end{array}$ & $\underset{\mathrm{c}}{0.63 \pm 0.02}$ & $\underset{\mathbf{c}}{0.71 \pm 0.02}$ & \\
\hline $\mathbf{A L U}$ & $0.01 \pm 0.00$ & $\begin{array}{c}0.09 \pm 0.02 \\
\mathrm{a}\end{array}$ & $0.26 \pm 0.02^{c}$ & $\underset{c}{0.44 \pm 0.02}$ & $0.01 \pm 0.01$ & $\underset{\mathrm{a}}{0.10 \pm 0.02}$ & $\underset{\mathrm{c}}{0.23 \pm 0.02}$ & $0.40 \pm 0.02^{\mathrm{c}}$ & $0.01 \pm 0.00$ & $\underset{\mathrm{a}}{0.09 \pm 0.03}$ & $\underset{\mathrm{c}}{0.25 \pm 0.02}$ & $\underset{c}{0.43 \pm 0.02}$ & \\
\hline AZI & $0.00 \pm 0.00$ & $0.11 \pm 0.02$ & $0.20 \pm 0.02$ & $\begin{array}{c}0.33 \pm 0.03 \\
\mathrm{a}\end{array}$ & $0.00 \pm 0.00$ & $0.10 \pm 0.02$ & $0.19 \pm 0.02$ & $0.29 \pm 0.01^{\mathrm{a}}$ & $0.00 \pm 0.00$ & $0.10 \pm 0.01$ & $0.19 \pm 0.03$ & $\begin{array}{c}0.28 \pm 0.03 \\
\mathrm{a}\end{array}$ & \multirow{3}{*}{ 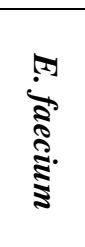 } \\
\hline $\mathrm{H}_{2} \mathrm{O}_{2}$ & $0.52 \pm 0.01$ & $\underset{\mathrm{a}}{0.61 \pm 0.01}$ & $0.73 \pm 0.01^{\mathrm{c}}$ & $\underset{\mathbf{c}}{0.87 \pm 0.01}$ & $0.52 \pm 0.01$ & $\underset{\mathrm{a}}{0.61 \pm 0.01}$ & $\underset{\mathbf{c}}{0.71 \pm 0.01}$ & $0.86 \pm 0.00^{c}$ & $0.51 \pm 0.01$ & $\begin{array}{c}0.60 \pm 0.01 \\
\mathrm{a}\end{array}$ & $\underset{\mathbf{c}}{0.70 \pm 0.01}$ & $\underset{\mathbf{c}}{0.82 \pm 0.01}$ & \\
\hline $\mathbf{A L U}$ & $0.00 \pm 0.00$ & $0.02 \pm 0.02$ & $0.18 \pm 0.02^{\mathfrak{c}}$ & $\begin{array}{c}0.42 \pm 0.02 \\
\mathbf{c}\end{array}$ & $0.00 \pm 0.00$ & $0.03 \pm 0.01$ & $0.17 \pm 0.02$ & $0.42 \pm 0.02^{\mathrm{c}}$ & $0.01 \pm 0.00$ & $0.03 \pm 0.01$ & $\begin{array}{c}0.16 \pm 0.02 \\
\mathbf{c}\end{array}$ & $\begin{array}{c}0.39 \pm 0.02 \\
\mathbf{c}\end{array}$ & \\
\hline AZI & $0.00 \pm 0.00$ & $\underset{\mathrm{c}}{0.10 \pm 0.02}$ & $0.26 \pm 0.00^{\mathbf{b}}$ & $\underset{\mathrm{c}}{0.34 \pm 0.03}$ & $0.00 \pm 0.00$ & $\begin{array}{c}0.09 \pm 0.01 \\
\mathrm{a}\end{array}$ & $\underset{\mathrm{a}}{0.24 \pm 0.01}$ & $0.31 \pm 0.03^{c}$ & $0.00 \pm 0.00$ & $\underset{\mathbf{b}}{0.09 \pm 0.01}$ & $0.3 \pm 0.01^{\mathrm{a}}$ & $\underset{\mathbf{b}}{0.31 \pm 0.03}$ & \multirow{3}{*}{ 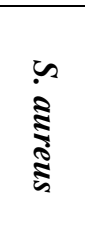 } \\
\hline $\mathrm{H}_{2} \mathrm{O}_{2}$ & $0.42 \pm 0.01$ & $\begin{array}{c}0.58 \pm 0.01 \\
\mathbf{c}\end{array}$ & $0.71 \pm 0.02^{\mathrm{c}}$ & $\underset{\mathbf{c}}{0.89 \pm 0.02}$ & $0.42 \pm 0.01$ & $\underset{\mathbf{c}}{0.58 \pm 0.01}$ & $\underset{\mathbf{c}}{0.70 \pm 0.01}$ & $\begin{array}{c}0.87 \pm 0.01 \\
\mathbf{c}\end{array}$ & $0.41 \pm 0.01$ & $\begin{array}{c}0.54 \pm 0.01 \\
\mathrm{a}\end{array}$ & $\underset{\mathbf{c}}{0.66 \pm 0.02}$ & $\underset{\mathbf{c}}{0.80 \pm 0.02}$ & \\
\hline $\mathbf{A L U}$ & $0.01 \pm 0.00$ & $\begin{array}{c}0.12 \pm 0.02 \\
\mathrm{a}\end{array}$ & $0.23 \pm 0.02^{\mathrm{c}}$ & $\underset{\mathbf{c}}{0.46 \pm 0.02}$ & $0.01 \pm 0.00$ & $\begin{array}{c}0.11 \pm 0.03 \\
\mathrm{a}\end{array}$ & $\begin{array}{c}0.23 \pm 0.02 \\
\mathbf{c}\end{array}$ & $\begin{array}{c}0.43 \pm 0.02 \\
\mathbf{c}\end{array}$ & $0.01 \pm 0.01$ & $\begin{array}{c}0.09 \pm 0.02 \\
\text { a }\end{array}$ & $\underset{\mathrm{c}}{0.21 \pm 0.01}$ & $\begin{array}{c}0.42 \pm 0.02 \\
\text { c }\end{array}$ & \\
\hline AZI & $1.09 \pm 0.03$ & $\underset{\mathbf{b}}{1.29 \pm 0.02}$ & $1.42 \pm 0.01^{\mathrm{c}}$ & $\underset{\mathrm{c}}{1.56 \pm 0.01}$ & $1.02 \pm 0.02$ & $\begin{array}{c}1.24 \pm 0.02 \\
\mathrm{a}\end{array}$ & $\underset{\mathbf{b}}{1.36 \pm 0.02}$ & $\underset{\mathrm{c}}{1.52 \pm 0.01}$ & $1.04 \pm 0.00$ & $\begin{array}{c}1.24 \pm 0.02 \\
\mathrm{a}\end{array}$ & $\underset{\text { c }}{1.38 \pm 0.03}$ & $\underset{\mathbf{c}}{1.50 \pm 0.04}$ & \multirow{3}{*}{$\begin{array}{l}\sqrt[\pi]{1} \\
\vdots \\
\vdots\end{array}$} \\
\hline $\mathbf{H}_{2} \mathbf{O}_{2}$ & $0.65 \pm 0.01$ & $\underset{\mathbf{b}}{0.81 \pm 0.02}$ & $0.98 \pm 0.02^{\mathrm{c}}$ & $\underset{\mathrm{c}}{1.13 \pm 0.02}$ & $0.64 \pm 0.01$ & $\underset{\mathbf{b}}{0.80 \pm 0.01}$ & $\underset{\mathbf{c}}{0.96 \pm 0.01}$ & $\underset{\mathbf{c}}{1.11 \pm 0.01}$ & $0.64 \pm 0.01$ & $\underset{\mathbf{c}}{0.79 \pm 0.01}$ & $\underset{\mathbf{c}}{0.96 \pm 0.02}$ & $\underset{\mathbf{c}}{1.11 \pm 0.02}$ & \\
\hline ALU & $0.01 \pm 0.01$ & $\begin{array}{c}0.13 \pm 0.03 \\
\mathrm{a}\end{array}$ & $0.26 \pm 0.02^{c}$ & $\underset{\mathbf{c}}{0.43 \pm 0.03}$ & $0.01 \pm 0.00$ & $\underset{\mathbf{a}}{0.11 \pm 0.02}$ & $\underset{\mathbf{c}}{0.25 \pm 0.02}$ & $\underset{\mathbf{c}}{0.42 \pm 0.02}$ & $0.01 \pm 0.01$ & $\begin{array}{c}0.09 \pm 0.02 \\
\mathrm{a}\end{array}$ & $\underset{\mathbf{c}}{0.21 \pm 0.02}$ & $\underset{\mathbf{c}}{0.40 \pm 0.01}$ & \\
\hline AZI & $1.10 \pm 0.03$ & $\begin{array}{c}1.23 \pm 0.02 \\
\mathrm{a}\end{array}$ & $1.38 \pm 0.02^{\mathrm{c}}$ & $\underset{\text { c }}{1.49 \pm 0.02}$ & $1.04 \pm 0.01$ & $\begin{array}{c}1.19 \pm 0.03 \\
\mathrm{a}\end{array}$ & $\underset{\mathbf{b}}{1.33 \pm 0.01}$ & $1.46 \pm 0.03^{\mathrm{c}}$ & $0.10 \pm 0.03$ & $\begin{array}{c}1.18 \pm 0.04 \\
\mathrm{a}\end{array}$ & $\begin{array}{c}1.30 \pm 0.02 \\
\mathbf{b}\end{array}$ & $\underset{\mathbf{b}}{1.43 \pm 0.02}$ & \multirow{3}{*}{ 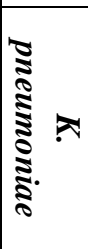 } \\
\hline $\mathrm{H}_{2} \mathrm{O}_{2}$ & $0.58 \pm 0.02$ & $\underset{\mathbf{c}}{0.71 \pm 0.02}$ & $0.82 \pm 0.01^{\mathrm{c}}$ & $\underset{\mathbf{c}}{1.00 \pm 0.02}$ & $0.57 \pm 0.02$ & $\underset{\mathrm{c}}{0.69 \pm 0.00}$ & $\underset{\mathbf{c}}{0.81 \pm 0.01}$ & $\underset{\mathbf{c}}{0.99 \pm 0.02}$ & $0.42 \pm 0.01$ & $\begin{array}{c}0.55 \pm 0.02 \\
\mathrm{a}\end{array}$ & $\underset{\mathrm{c}}{0.66 \pm 0.01}$ & $\underset{\mathbf{c}}{0.81 \pm 0.03}$ & \\
\hline ALU & $0.03 \pm 0.00$ & $\begin{array}{c}0.11 \pm 0.02 \\
\text { a }\end{array}$ & $0.26 \pm 0.02^{\mathrm{c}}$ & $\underset{\mathrm{c}}{0.49 \pm 0.02}$ & $0.02 \pm 0.00$ & $0.1 \pm 0.03$ & $\underset{\mathrm{b}}{0.29 \pm 0.02}$ & $\begin{array}{c}0.47 \pm 0.02 \\
\mathrm{c}\end{array}$ & $\begin{array}{c}0.01 \pm \\
0.00\end{array}$ & $0.07 \pm 0.02$ & $0.21 \pm 0.03$ & $\begin{array}{c}0.45 \pm 0.03 \\
\mathrm{c}\end{array}$ & \\
\hline
\end{tabular}


Similarly, alum concentrations, $35 \mathrm{mg} / \mathrm{ml}$, $17.5 \mathrm{mg} / \mathrm{ml}, \quad 8.7 \mathrm{mg} / \mathrm{ml}$ and $4.4 \mathrm{mg} / \mathrm{ml}$ showed $0.438 \mathrm{~cm}, 0.263 \mathrm{~cm}, 0.089 \mathrm{~cm}$, $0.005 \mathrm{~cm}$ zones of inhibition against $E$. faecalis, $0.421 \mathrm{~cm}, 0.184 \mathrm{~cm}, 0.019 \mathrm{~cm}, 0 \mathrm{~cm}$ zones of inhibition against E. faecium, $0.455 \mathrm{~cm}, \quad 0.234 \mathrm{~cm}, \quad 0.122 \mathrm{~cm}, \quad 0.011 \mathrm{~cm}$ zones of inhibition against $S$. aureus, $0.430 \mathrm{~cm}, \quad 0.258 \mathrm{~cm}, \quad 0.126 \mathrm{~cm}, \quad 0.013 \mathrm{~cm}$ zones of inhibition against $E$. coli, and $0.485 \mathrm{~cm}, 0.264 \mathrm{~cm}, 0.106 \mathrm{~cm}, 0.025 \mathrm{~cm}$ zones of inhibition against $K$. pneumoniae, respectively at $24 \mathrm{hr}$ incubation (Table 1 ). These results concur with the previous findings which reported protective effect of the 100 PPM alum solution as less than 1000 PPM alum or more [37]. In 2014, Bnyan et al [38], additionally observed a paramount bactericidal effect of alum however, the mechanism of bactericidal effect of alum is not prominent [39]. Some postulations attribute the antibacterial effect of alum to reduction in acidity or deleterious effects on bacterial cell wall [40].

Thus, $\mathrm{H}_{2} \mathrm{O}_{2}$ bacterial susceptibility trend was $E$. coli $>K$. pneumoniae $>S$. aureus $>E$. faecium $>E$. faecalis and ALM susceptibility trend were as $K$. pneumoniae $>S$. aureus $>E$. faecalis $>E$. coli $>$ E. faecium at highest tested concentration and $24 \mathrm{hr}$ of incubation period (Table 1). However, $\mathrm{H}_{2} \mathrm{O}_{2}$ showed more zone of inhibition than alum against all tested bacteria at all tested concentrations and incubation. Interestingly, zones were higher at lower incubation time which reduced later in the order of $24 \mathrm{hr}>48 \mathrm{hr}$ $>72 \mathrm{hr}$ incubation times even at same $\mathrm{H}_{2} \mathrm{O}_{2}$ and ALM concentration (Table 1).
Similarly, Saranraj et al., 2012, [31] reported hydrogen peroxide as an effective disinfectant but this effect was a short term which means hydrogen peroxide has no long-term or preserving effect. Similar, incubation effect was observed for azithromycin (AZI), used as a positive control as shown in (Table 1). However, both $\mathrm{H}_{2} \mathrm{O}_{2}$ and ALM showed more zones of inhibition at the highest tested concentration $(35 \mathrm{mg} / \mathrm{ml})$ than AZI against E. faecalis, E. faecium and $S$. aureus at $24 \mathrm{hr}, 48 \mathrm{hr}$ and $72 \mathrm{hr}$ of incubation.

\section{Combined antibacterial effect of alum and hydrogen peroxide}

To check combination effect, alum and hydrogen peroxide were used in 1:1 ratio with two-fold increase in concentration from well-1 to well-4 in well diffusion method, respectively, as explained in method section. The combination of alum and hydrogen peroxide in $1: 1$ ratio forming $4.4 \mathrm{mg} / \mathrm{ml}, 8.7 \mathrm{mg} / \mathrm{ml}, 17.5 \mathrm{mg} / \mathrm{ml}$ and $35 \mathrm{mg} / \mathrm{ml}$ total concentrations showed $0.508 \mathrm{~cm}, 0.635 \mathrm{~cm}, 0.779 \mathrm{~cm}$ and $1.052 \mathrm{~cm}$ zones of inhibition against E. faecalis, $0.497 \mathrm{~cm}, 0.656 \mathrm{~cm}, 0.802 \mathrm{~cm}$ and $0.979 \mathrm{~cm}$ zones of inhibition against E. faecium, $0.406 \mathrm{~cm}, 0.552 \mathrm{~cm}, 0.672 \mathrm{~cm}$ and $0.843 \mathrm{~cm}$ zones of inhibition against $S$. aureus, $0.414 \mathrm{~cm}, 0.547 \mathrm{~cm}, 0.735 \mathrm{~cm}$ and $0.870 \mathrm{~cm}$ zones of inhibition against $E$. coli and, $0.397 \mathrm{~cm}, 0.575 \mathrm{~cm}, 0.770 \mathrm{~cm}$ and $0.968 \mathrm{~cm}$ zones of inhibition against $K$. pneumoniae, respectively at $24 \mathrm{hr}$ of inhibition (Table 2 ). In combination test, zones were higher at lower incubation time which reduced later in order of $24 \mathrm{hr}>48 \mathrm{hr}>72 \mathrm{hr}$ incubation times same as $\mathrm{H}_{2} \mathrm{O}_{2}$ and ALM in individual test. 
Table 2. Antibacterial susceptibility of selected bacteria against $\mathrm{H}_{2} \mathrm{O}_{2}$ and ALM in combination (1:1) at indicated concentrations and incubation periods. Student's t-test was applied to check significance where ${ }^{\mathrm{a} p}<0.05 ;{ }^{b} \mathbf{p}<0.005,{ }^{\mathrm{c}} \mathbf{p}<0.0001$

\begin{tabular}{|c|c|c|c|c|c|c|c|c|c|c|c|c|c|}
\hline \multirow{3}{*}{$=$} & \multicolumn{4}{|c|}{ Concentration $(\mathrm{mg} / \mathrm{ml})$} & \multicolumn{4}{|c|}{ Concentration (mg/ml) } & \multicolumn{4}{|c|}{ Concentration $(\mathrm{mg} / \mathrm{ml})$} & \multirow{3}{*}{$\stackrel{2}{2}$} \\
\hline & 4.4 & 8.7 & $\mathbf{1 7 . 5}$ & 35 & 4.4 & 8.7 & 17.5 & 35 & 4.4 & 8.7 & 17.5 & 35 & \\
\hline & \multicolumn{4}{|c|}{$\begin{array}{l}\text { Zone of inhibition }(\mathrm{cm}) \\
\text { at } 24 \mathrm{hr} \text { incubation }\end{array}$} & \multicolumn{4}{|c|}{$\begin{array}{l}\text { Zone of inhibition }(\mathrm{cm}) \\
\text { at } 48 \mathrm{hr} \text { incubation }\end{array}$} & \multicolumn{4}{|c|}{$\begin{array}{l}\text { Zone of inhibition }(\mathrm{cm}) \\
\text { at } 72 \mathrm{hr} \text { incubation }\end{array}$} & \\
\hline 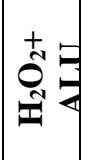 & $0.51 \pm 0.02$ & $0.64 \pm 0.01^{\mathrm{a}}$ & $0.78 \pm 0.01^{\mathrm{c}}$ & $\underset{\mathrm{c}}{1.05 \pm 0.02}$ & $0.46 \pm 0.02$ & $\underset{\mathbf{a}}{0.57 \pm 0.02}$ & $\underset{\mathrm{c}}{0.68 \pm 0.02}$ & $\underset{\mathrm{c}}{0.92 \pm 0.00}$ & $0.45 \pm 0.05$ & $0.53 \pm 0.02$ & $\underset{\mathrm{a}}{0.64 \pm 0.02}$ & $\underset{\mathbf{c}}{0.88 \pm 0.02}$ & 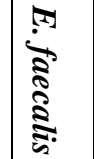 \\
\hline 声目 & $0.50 \pm 0.05$ & $0.66 \pm 0.06^{\mathrm{a}}$ & $0.81 \pm 0.03^{\mathbf{b}}$ & $\underset{\text { c }}{0.98 \pm 0.04}$ & $0.48 \pm 0.03$ & $\underset{\mathrm{a}}{0.63 \pm 0.06}$ & $\underset{\mathbf{c}}{0.75 \pm 0.04}$ & $\underset{\mathbf{c}}{0.91 \pm 0.02}$ & $0.48 \pm 0.04$ & $\underset{\mathrm{a}}{0.62 \pm 0.04}$ & $\underset{\mathrm{c}}{0.73 \pm 0.03}$ & $\underset{\mathbf{c}}{0.88 \pm 0.03}$ & 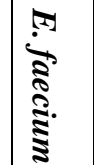 \\
\hline $\begin{array}{l}+ \\
0 \\
0 \\
\mathbf{I}\end{array}$ & $0.41 \pm 0.02$ & $0.55 \pm 0.03^{\mathrm{a}}$ & $0.67 \pm 0.02^{\mathbf{b}}$ & $\underset{\text { c }}{0.84 \pm 0.02}$ & $0.38 \pm 0.03$ & $0.51 \pm 0.03$ & $\underset{\mathbf{b}}{0.61 \pm 0.01}$ & $\underset{\mathbf{c}}{0.78 \pm 0.01}$ & $0.39 \pm 0.02$ & $0.51 \pm 0.02$ & $\underset{\mathrm{a}}{0.60 \pm 0.02}$ & $\underset{\mathbf{c}}{0.76 \pm 0.01}$ & 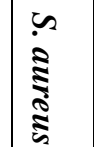 \\
\hline 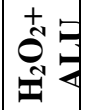 & $0.41 \pm 0.01$ & $0.55 \pm 0.01$ & $0.74 \pm 0.01^{\mathrm{c}}$ & $\underset{\mathbf{c}}{0.87 \pm 0.03}$ & $0.37 \pm 0.01$ & $0.49 \pm 0.01$ & $\begin{array}{c}0.66 \pm 0.01 \\
2^{c}\end{array}$ & $\underset{\mathrm{c}}{0.79 \pm 0.02}$ & $0.39 \pm 0.01$ & $\frac{0.53 \pm 0.01}{\mathrm{a}}$ & $\underset{\mathbf{c}}{0.68 \pm 0.01}$ & $\underset{\mathbf{c}}{0.78 \pm 0.03}$ & $\begin{array}{l}1 \\
\vdots \\
\vdots\end{array}$ \\
\hline 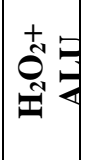 & $0.40 \pm 0.03$ & $\underset{\mathbf{c}}{0.58 \pm 0.04}$ & $0.77 \pm 0.02^{c}$ & $\underset{\mathrm{c}}{0.97 \pm 0.02}$ & $0.37 \pm 0.04$ & $\underset{\mathrm{a}}{0.50 \pm 0.04}$ & $\underset{\mathrm{c}}{0.69 \pm 0.02}$ & $\begin{array}{c}0.86 \pm 0.01 \\
1^{\mathrm{c}}\end{array}$ & $0.36 \pm 0.02$ & $\begin{array}{c}0.50 \pm 0.03 \\
\mathbf{a}\end{array}$ & $\underset{c}{0.69 \pm 0.03}$ & $\underset{\mathbf{c}}{0.83 \pm 0.01}$ & 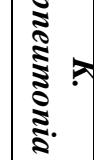 \\
\hline
\end{tabular}


Interestingly, $\mathrm{H}_{2} \mathrm{O}_{2}$ and ALM in combination showed more zones of inhibition at the highest tested concentration $(35 \mathrm{mg} / \mathrm{ml})$ than AZI against E. faecalis, E. faecium and $S$. aureus at $24 \mathrm{hr}, 48 \mathrm{hr}$ and $72 \mathrm{hr}$ of incubation (Table 1 \& 2). Moreover, $\mathrm{H}_{2} \mathrm{O}_{2}$ and ALM in the combination showed more zone of inhibition than $\mathrm{H}_{2} \mathrm{O}_{2}$ alone against $E$. faecalis and E. faecium and, also more effect than ALM alone against all tested bacteria $i ; e$ E. faecalis, E. faecium, $S$. aureus, E. coli and $K$. pneumoniae at all incubations (Table $1 \& 2$ ), summarized in (Table 3). Amadi et al. [30] found similarly increased antibacterial impacts of alum with guava leaf extract in combination. Another study reported that the combination of alum with antibiotics (tetracycline and cefotaxime) was more effective than antibiotic combination and the combination effect was more efficient than an individual effect [40]. Similarly, hydrogen peroxide in combination with lactoperoxidase and thiocyanate is reported much more effective than hydrogen peroxide alone as an inhibitor of bacterial metabolism and growth [41]. Thus, the combination strategy provides us with an opportunity to improve their efficacy at low concentration. Therefore, side effects associated with a higher load of drugs can be reduced without compromising their efficacy [42]. Due to these advantages, combination therapies have become a standard in many areas including cancer treatment [43], hypertension [44], asthma [45], and AIDS $[46,47]$ which open a new horizon for research and new hope for disease control including bacterial infections in future.

Table 3. Comparison of antibacterial effect at highest tested concentration $(35 \mathrm{mg} / \mathrm{ml})$ of test compounds and their combinations against selected bacteria

\begin{tabular}{|c|l|l|}
\hline Bacteria & Susceptibility trend against tested compounds \\
\hline E. faecalis & $\mathrm{H}_{2} \mathrm{O}_{2}+\mathrm{ALU}>\mathrm{H}_{2} \mathrm{O}_{2}>\mathrm{ALU}>\mathrm{AZI}$ \\
\hline $\boldsymbol{E}$. faecium & $\mathrm{H}_{2} \mathrm{O}_{2}+\mathrm{ALU}>\mathrm{H}_{2} \mathrm{O}_{2}>\mathrm{ALU}>\mathrm{AZI}$ \\
\hline S. aureus & $\mathrm{H}_{2} \mathrm{O}_{2}>\mathrm{H}_{2} \mathrm{O}_{2}+\mathrm{ALU}>\mathrm{ALU}>\mathrm{AZI}$ \\
\hline E. coli & $\mathrm{AZI}>\mathrm{H}_{2} \mathrm{O}_{2}>\mathrm{H}_{2} \mathrm{O}_{2}+\mathrm{ALU}>\mathrm{ALU}$ \\
\hline K. pneumoniae & $\mathrm{AZI}>\mathrm{H}_{2} \mathrm{O}_{2}>\mathrm{H}_{2} \mathrm{O}_{2}+\mathrm{ALU}>\mathrm{ALU}$ \\
\hline
\end{tabular}

\section{Conclusion}

This study found that the increasing concentration of $\mathrm{H}_{2} \mathrm{O}_{2}$ and ALM have increasing antibacterial effects on tested bacteria which reduced with time. Moreover, $\mathrm{H}_{2} \mathrm{O}_{2}$ and ALM in combination showed increased zone inhibition than ALM alone (against all bacteria) and $\mathrm{H}_{2} \mathrm{O}_{2}$ alone (against E. faecalis and E. faecium) without increasing final concentration. Thus, a combination strategy might help us in minimizing dose-dependent side effects of drugs without compromising effectiveness.

\section{Authors' contributions}

Conceived and designed the experiments: A Ali \& H Khurshid, Performed the experiments: H Khurshid \& A Ali, Analyzed the data: A Ali \& B Akbar,
Contributed materials/ analysis/ tools: $\mathrm{M}$ Rafiq, F Nazir \& M Ahmed, Wrote the paper: I Ali \& M Ahmed.

\section{Acknowledgement}

This work was financially supported by Mirpur University of Science \& Technology (MUST), AJK, Pakistan. The authors are thankful to Combined Military Hospital (CMH) Rawalpindi (Pakistan) for providing us bacterial isolates for this study. The authors declare no financial or any other conflict of interest.

\section{References}

1. McDonnell G \& Russell AD (1999). Antiseptics and disinfectants: activity, action, and resistance. Clin Microbiol Rev 12(1): 147-179.

2. Bowler PG, Duerden BI \& Armstrong DG (2001). Wound microbiology and 
associated approaches to wound management. Clin Microbiol Rev 14(2): $244-269$.

3. Foucquier J \& Guedj M (2015). Analysis of drug combinations: current methodological landscape. Pharma Res Per 3(3): e00149.

4. Juven JB \& Pierson MD (1996). Antibacterial effects of hydrogen peroxide and methods for its detection and quantitationt. $J$ of Food Prot 59(11): 1233-1241.

5. Prabhu R, Kohale B, Agrawal AA, Wagle SV, Bhartiya G \& Chaudhari D (2017). A comparative clinical study to evaluate the effect of $1.5 \%$ hydrogen peroxide mouthwash as an adjunct to $0.2 \%$ chlorhexidine mouthwash to reduce dental stains and plaque formation. IJCMR 4(10): 21812184.

6. Presterl E, Suchomel M, Eder M, Reichmann S, Lassnigg A, Graninger W \& Rotter M (2007). Effects of alcohols, povidone-iodine and hydrogen peroxide on biofilms of Staphylococcus epidermidis. $J$ Antimicrob Chemother 60(2): 417420.

7. Atakan IH, Kaplan M, Kaya E, Aktoz T \& Inci O (2002). A life-threatening infection: Fournier's gangrene. Int Urol Nephrol 34(3): 387-392.

8. Malik DJ, Shaw CM, Rielly CD \& Shama G (2013). The inactivation of Bacillus subtilis spores at low concentrations of hydrogen peroxide vapour. J Food Eng 114(3): 391-396.

9. Arndt RE, Wagner EJ \& Routledge MD (2001). Reducing or withholding hydrogen peroxide treatment during a critical stage of rainbow trout development: effects on eyed eggs, hatch, deformities, and fungal control. North Am J Aquaculture 63(2): 161166.

10. Wagner EJ, Oplinger RW \& Bartley RWM (2012). Laboratory and production scale disinfection of salmonid eggs with hydrogen peroxid. North Am J Aquaculture 74(1): 92-99.

11. Ukuku DO, Bari ML, Kawamoto S \& Isshiki K (2005). Use of hydrogen peroxide in combination with nisin, sodium lactate and citric acid for reducing transfer of bacterial pathogens from whole melon surfaces to fresh-cut pieces. Int $J$ Food Microbiol 104(2): 225-233.

12. Chang $\mathrm{R}$ (2005). Chemistry. 8th edition. McGraw-Hill Companies. Inc New York USA.

13. Olmez A, Can H, Ayhan $\mathrm{H}$ \& Olur $\mathrm{H}$ (1998). Effect of an alum-containing mouthrinse in children for plaque and salivary levels of selected oral microflora. J Clin Pediatr Dent 22(4): 335-440.

14. Bestoon MF (2012). Evidence of feasibility of aluminum potassium sulphate (alum) solution as a root canal irrigant. $J$ Bagh College Dentistry 24(1): 1-5.

15. Mourughan K \& Suryakanth MP (2004). Evaluation of an alumcontaining mouthrinse for inhibition of salivary Streptococcus mutans levels in children- A controlled clinical trial. J Indian Soc Ped Prev Dent 22(3): 100-105.

16. Alzormor AK, Moharram AS \& AlAbsi NM (2014). Formulation and evaluation of potash alum as deodorant lotion and after shaving astringent as cream and gel. Int Curr Pharm J 3(2): 228-233.

17. Potter NN \& Hotchkiss JH (2007). Food Science. 5th edition. CBS Publishers and Distributors, Daryaganji, New Delhi India.

18. Ahmed KT (2011). Inhibition of swarming in Proteus mirabilis by alum (hydrated aluminum potassium sulphate). $J$ University of Anbar for Pure Science 5(2): 20-24.

19. Humphrey TJ (1990). The synergistic inhibition of Campylobacter jejuni by rifampicin and hydrogen peroxide. Lett Appl Microbiol 10(2): 97-100. 
20. Steinberg D, Heling I, Daniel I \& Ginsburg I (1999). Antibacterial synergistic effect of chlorhexidine and hydrogen peroxide against Streptococcus sobrinus Streptococcus faecalis and Staphylococcus aureus. $J$ Oral Rehabil 26(2): 151-156.

21. Almeida CEB, Felicio DL, Galhardo RS, Cabral-Neto JB \& Leitao AC (1999). Synergistic lethal effect between hydrogen peroxide and neocuproine (2,9-dimethyl 1,10phenanthroline) in Escherichia coli. Mutat Res DNA Repair 433(1): 5966.

22. Carlsson J, Edlund MB \& Hanstrom L (1984). Bactericidal and cytotoxic effects of hypothiocyanite-hydrogen peroxide mixtures. Infect Immun 44(3): 581-586.

23. Miyasaki KT, Genco RJ \& Wilson ME (1986). Antimicrobial properties of hydrogen peroxide and sodium bicarbonate individually and in combination against selected oral, gram-negative, facultative bacteria. $J$ Dent Res 65(9): 1142-1148.

24. Voboril R \& Weberova J (2004). Successful treatment of infected vascular prosthetic grafts in the groin using conservative therapy with povidoneiodine solution. Ann Vasc Surg 18(3): 372-375.

25. Ulivieri S, Toninelli S, Petrini C, Giorgio A \& Oliveri G (2011). Prevention of postoperative infections in spine surgery by wound irrigation with a solution of povidone-iodine and hydrogen peroxide. Arch Orthop Trauma Surg 131(9): 1203-1206.

26. SM Ng \& Wieckowski S (1989). Stable hydrogen peroxide dental gel, US Patent-4839156 ColgatePalmolive Company. 1199.

27. Li Y \& Wu C (2013). Enhanced inactivation of Salmonella Typhimurium from blueberries by combinations of sodium dodecyl sulfate with organic acids or hydrogen peroxide. Food Res Int 54(2): $1553-$ 1559.

28. Martin H \& Maris P (2012). Synergism between hydrogen peroxide and seventeen acids against six bacterial strains. J Appl Microbiol 113(3): 578-590.

29. Shama G (1992). Inactivation of Escherichia coli by ultraviolet light and hydrogen peroxide in a thin film contactor. Lett Appl Microbiol 15(6): 259-260.

30. Amadi LO, Wanabia D \& Amadi V (2016). Combination effects of alum and guava (Psidium guajava) leaf extracts on some pathogens from clinical samples. Int J Curr Res 8(5): 31354-31358.

31. Cadnum JL, Mana TS, Jencson A, Thota P, Kundrapu S, Donskey CJ (2015). Effectiveness of a hydrogen peroxide spray for decontamination of soft surfaces in hospitals. Am J Infect Control43(12):1357-1359.

32. Juven BJ \& Pierson MD (1996). Antibacterial effects of hydrogen peroxide and methods for its detection and quantitation. J Food Prot 59(11): 1233-1241.

33. Uhl L, Gerstel A, Chabalier M \& Dukan S (2015). Hydrogen peroxide induced cell death: One or two modes of action? Heliyon 1(4): e00049.

34. Imlay JA (2003). Pathways of oxidative damage. Annu Rev Microbiol 57: 395-418.

35. Imlay JA \& Linn S (1986). Bimodal pattern of killing of DNA-repairdefective or anoxically grown Escherichia coli by hydrogen peroxide. J Bacteriol 166(2): 519527.

36. Imlay JA \& Linn S (1988). DNA damage and oxygen radical toxicity. Science 240(4857): 1302-1309.

37. Putt MS \& Kleber CJ (1995). Effect of aluminum concentration on dental caries formation in the rat. Oral Dis 1(2): 80-5. 
38. Bnyan IA, Alta'ee AH \& Kadhum NH (2014). Antibacterial activity of aluminum potassium sulfate and syzygium aromaticum extract against pathogenic microorganisms. J Nat Sci Res 4(15): 11-4.

39. Arul PF, Babu GD, Lavanya M, Vidhya KS \& Devasena T (2011). Toxicity studies of aluminium oxide nanoparticles in cell lines. Int $J$ Nanotechnol Appl 5(2): 99-107.

40. Ali ZM (2018). Synergistic antibacterial interaction between an alum and antibiotics on some microorganism. Sci J Med Res 2(5): 47-51.

41. Thomas EL, Milligan TW, Joyner RE \& Jefferson MM (1994). Antibacterial activity of hydrogen peroxide and the lactoperoxidase hydrogen peroxidethiocyanate system against oral streptococci. Infect Immun 62(2): 529535.

42. Danesh A, Ljungh $\AA$, Mattiasson B \& Mamo G (2016). Synergistic effect of haloduracin and chloramphenicol against clinically important grampositive bacteria. Biotechnol Rep 13: 37-41.

43. Humphrey RW, Brockway LM, Bonk DT, Dohoney KM, Doroshow JH \& Meech SJ (2011). Opportunities and challenges in the development of experimental drug combinations for cancer. J Natl Cancer Inst 103(16): 1222-1226.

44. Glass G (2004). Cardiovascular combinations. Nat Rev Drug Discov 3(9): 731-732.

45. Nelson HS (2001). Combination treatment with fluticasone propionate/salmeterol in the treatment of asthma, J Allergy Clin Immunol 107(2): 398-416.

46. Larder BA, Kemp SD \& Harrigan PR (1995). Potential mechanism for sustained antiretroviral efficacy of AZT-3TC combination therapy. Science 269(5224): 696-699.

47. Oversteegen L, Shah M \& Rovini H (2007). HIV combination products. Nat Rev Drug Discov 6(12): 951-959. 